\title{
Assessment of Undiscovered Oil and Gas Resources in Jurassic and Cretaceous Strata of the Gulf Coast, 2010
}

Using a geology-based assessment methodology, the U.S. Geological Survey estimated means of 147.4 trillion cubic feet of undiscovered natural gas, 2.4 billion barrels of undiscovered oil, and 2.96 billion barrels of undiscovered natural gas liquids in Jurassic and Cretaceous strata in onshore lands and State waters of the Gulf Coast.

\section{Introduction}

The U.S. Geological Survey (USGS) recently completed an assessment of the undiscovered oil and gas resources in Jurassic and Cretaceous strata of the onshore areas and State waters of the Gulf Coast (fig. 1). The assessment is based on geologic elements of a total petroleum system (TPS), including characterization of hydrocarbon source rocks (source-rock maturation, hydrocarbon generation and migration), reservoir rocks (sequence stratigraphy and petrophysical properties), and hydrocarbon traps (trap formation, timing, and seals). Using these criteria, the USGS defined an Upper JurassicCretaceous-Tertiary Composite TPS for conventional oil and gas resources that extends around the entire Gulf of Mexico, including portions of both the United States and Mexico. The present assessment of undiscovered conventional oil and gas resources includes only that portion of the TPS that lies onshore and in State waters of the United States (fig. 1). This area extends geographically from the southwest boundary of the Western Gulf Province (47) on the Texas-Mexico border

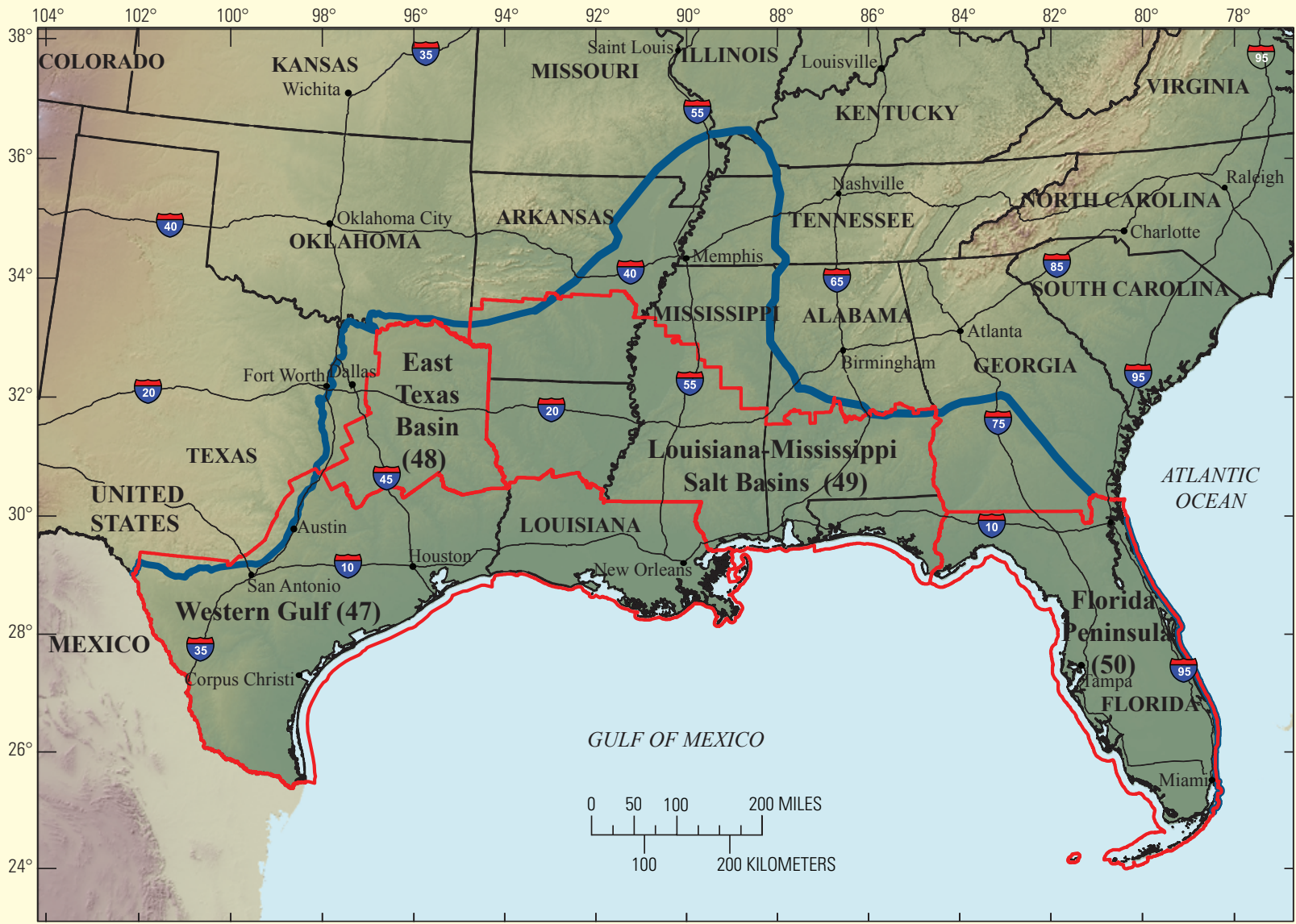

Figure 1. Map of the Gulf Coast region showing the part of the Upper Jurassic-Cretaceous-Tertiary Composite Total Petroleum System that is onshore and in State waters of the Gulf Coast of the United States (blue line indicates onshore boundary in the United States). Province boundaries defined by the U.S. Geological Survey are indicated by red outlines. 
Table 1. Gulf Coast assessment results.

[MMBO, million barrels of oil; BCFG, billion cubic feet of gas; MMBNGL, million barrels of natural gas liquids. Results shown are fully risked estimates. For gas accumulations, all liquids are included as NGL (natural gas liquids). F95 represents a 95 percent chance of at least the amount tabulated; other fractiles are defined similarly. Fractiles are additive under the assumption of perfect positive correlation. TPS, total petroleum system; AU, assessment unit. Gray shading indicates not applicable]

\begin{tabular}{|c|c|c|c|c|c|c|c|c|c|c|c|c|c|}
\hline \multirow{3}{*}{$\begin{array}{c}\text { Total Petroleum Systems } \\
\text { (TPS) } \\
\text { and Assessment Units (AU) }\end{array}$} & \multirow{3}{*}{$\begin{array}{l}\text { Field } \\
\text { Type }\end{array}$} & \multicolumn{12}{|c|}{ Total Undiscovered Resources } \\
\hline & & \multicolumn{4}{|c|}{ Oil (MMBO) } & \multicolumn{4}{|c|}{ Gas (BCFG) } & \multicolumn{4}{|c|}{ NGL (MMBNGL) } \\
\hline & & F95 & F50 & F5 & Mean & F95 & F50 & F5 & Mean & F95 & F50 & F5 & Mean \\
\hline \multicolumn{14}{|c|}{ Upper Jurassic-Creataceous-Tertiary Composite TPS (50490100) } \\
\hline \multirow{2}{*}{$\begin{array}{l}\text { Norphlet Salt Basins } \\
\text { and Updip AU (50490102) }\end{array}$} & Oil & 13 & 48 & 106 & 53 & 8 & 31 & 73 & 34 & 1 & 2 & 6 & 3 \\
\hline & Gas & & & & & 371 & 1,579 & 3,709 & 1,758 & 12 & 53 & 135 & 61 \\
\hline \multirow{2}{*}{$\begin{array}{l}\text { Norphlet Mobile Bay } \\
\text { Deep Gas AU (50490103) }\end{array}$} & Oil & 0 & 0 & 0 & 0 & 0 & 0 & 0 & 0 & 0 & 0 & 0 & 0 \\
\hline & Gas & & & & & 208 & 1,175 & 4,029 & 1,514 & 0 & 2 & 7 & 3 \\
\hline \multirow{2}{*}{$\begin{array}{l}\text { Norphlet South Texas Gas AU } \\
\quad(50490104)\end{array}$} & Oil & 0 & 0 & 0 & 0 & 0 & 0 & 0 & 0 & 0 & 0 & 0 & 0 \\
\hline & Gas & & & & & 0 & 153 & 748 & 233 & 0 & 5 & 27 & 8 \\
\hline \multirow{2}{*}{$\begin{array}{r}\text { Smackover Updip and Peripher- } \\
\text { al Fault Zone AU (50490105) }\end{array}$} & Oil & 27 & 76 & 136 & 78 & 29 & 85 & 169 & 90 & 2 & 7 & 15 & 7 \\
\hline & Gas & & & & & 46 & 151 & 304 & 160 & 4 & 15 & 32 & 16 \\
\hline \multirow{2}{*}{$\begin{array}{l}\text { Smackover Salt Basin AU } \\
\quad(50490106)\end{array}$} & Oil & 21 & 75 & 149 & 79 & 25 & 90 & 199 & 98 & 2 & 7 & 17 & 8 \\
\hline & Gas & & & & & 265 & 889 & 1,693 & 926 & 32 & 112 & 235 & 120 \\
\hline \multirow{2}{*}{$\begin{array}{l}\text { Smackover South Texas AU } \\
\quad(50490107)\end{array}$} & Oil & 6 & 22 & 51 & 25 & 7 & 25 & 62 & 28 & 1 & 2 & 5 & 2 \\
\hline & Gas & & & & & 203 & 699 & 1,419 & 744 & 19 & 68 & 151 & 74 \\
\hline \multirow{2}{*}{$\begin{array}{l}\text { Haynesville Western Shelf- } \\
\text { Sabine Platform Carbonate } \\
\text { Gas AU (50490116) }\end{array}$} & Oil & 0 & 0 & 0 & 0 & 0 & 0 & 0 & 0 & 0 & 0 & 0 & 0 \\
\hline & Gas & & & & & 143 & 541 & 1,180 & 587 & 2 & 8 & 19 & 9 \\
\hline \multirow{2}{*}{$\begin{array}{l}\text { Haynesville Shelf Carbonate } \\
\text { and Sandstone Oil and Gas } \\
\text { AU (50490117) }\end{array}$} & Oil & 7 & 27 & 64 & 30 & 9 & 37 & 95 & 43 & 1 & 3 & 9 & 4 \\
\hline & Gas & & & & & 9 & 27 & 75 & 32 & 0 & 1 & 3 & 1 \\
\hline \multirow{2}{*}{$\begin{array}{l}\text { Bossier East Texas Basin } \\
\text { Sandstone Gas AU (50490118) }\end{array}$} & Oil & 0 & 0 & 0 & 0 & 0 & 0 & 0 & 0 & 0 & 0 & 0 & 0 \\
\hline & Gas & & & & & 590 & 2,512 & 5,806 & 2,765 & 8 & 34 & 86 & 39 \\
\hline \multirow{2}{*}{$\begin{array}{l}\text { Bossier Louisiana-Mississippi } \\
\text { Shelf Edge Sandstone Gas } \\
\text { AU (50490119) }\end{array}$} & Oil & 0 & 0 & 0 & 0 & 0 & 0 & 0 & 0 & 0 & 0 & 0 & 0 \\
\hline & Gas & & & & & 211 & 945 & 2,368 & 1,072 & 3 & 13 & 35 & 15 \\
\hline \multirow{2}{*}{$\begin{array}{l}\text { Knowles-Calvin Gas AU } \\
\quad(50490120)\end{array}$} & Oil & 0 & 0 & 0 & 0 & 0 & 0 & 0 & 0 & 0 & 0 & 0 & 0 \\
\hline & Gas & & & & & 621 & 3,069 & 7,382 & 3,423 & 6 & 30 & 78 & 34 \\
\hline \multirow{2}{*}{$\begin{array}{l}\text { Sligo-James Carbonate Platform } \\
\text { Gas and Oil AU (50490121) }\end{array}$} & Oil & 11 & 45 & 106 & 50 & 31 & 132 & 336 & 151 & 1 & 6 & 16 & 7 \\
\hline & Gas & & & & & 167 & 587 & 1,279 & 640 & 5 & 17 & 41 & 19 \\
\hline \multirow{2}{*}{$\begin{array}{l}\text { Lower Cretaceous Basinal } \\
\text { Gas AU (50490122) }\end{array}$} & & \multirow{2}{*}{\multicolumn{4}{|c|}{ Not quantitatively assessed }} & & & & & & & & \\
\hline & Gas & & & & & & & & & & & & \\
\hline Sligo Sandstone Gas and Oil & Oil & 3 & 9 & 19 & 10 & 1 & 4 & 9 & 4 & 0 & 0 & 0 & 0 \\
\hline AU (50490123) & Gas & & & & & 140 & 440 & 803 & 453 & 1 & 4 & 9 & 5 \\
\hline Greater Glen Rose Carbonate & Oil & 23 & 71 & 153 & 78 & 78 & 260 & 602 & 290 & 4 & 13 & 32 & 15 \\
\hline $\begin{array}{l}\text { Shelf and Reef Gas and Oil } \\
\text { AU (50490124) }\end{array}$ & Gas & & & & & 252 & 720 & 1,480 & 778 & 7 & 22 & 50 & 25 \\
\hline Albion Clactio $\mathrm{UU}(50400125)$ & Oil & 11 & 35 & 69 & 37 & 9 & 31 & 67 & 33 & 0 & 1 & 3 & 1 \\
\hline Albian Clastic AU (50490125) & Gas & & & & & 39 & 116 & 207 & 119 & 1 & 3 & 6 & 3 \\
\hline Updip Albian Clastic AU & Oil & 0 & 1 & 3 & 1 & 0 & 0 & 3 & 1 & 0 & 0 & 0 & 0 \\
\hline$(50490126)$ & Gas & & & & & 0 & 3 & 15 & 4 & 0 & 0 & 0 & 0 \\
\hline Fredericksburg-Buda Carbonate & Oil & 10 & 37 & 83 & 40 & 26 & 100 & 246 & 113 & 1 & 5 & 12 & 5 \\
\hline $\begin{array}{l}\text { Platform-Reef Gas and Oil } \\
\text { AU (50490127) }\end{array}$ & Gas & & & & & 120 & 466 & 1,041 & 509 & 2 & 8 & 20 & 9 \\
\hline Eagle Ford Updip Sandstone Oil & Oil & 41 & 136 & 253 & 141 & 53 & 184 & 381 & 197 & 1 & 4 & 8 & 4 \\
\hline and Gas AU (50490128) & Gas & & & & & 86 & 289 & 571 & 305 & 3 & 11 & 24 & 12 \\
\hline
\end{tabular}


Table 1. Gulf Coast assessment results.-Continued

[MMBO, million barrels of oil; BCFG, billion cubic feet of gas; MMBNGL, million barrels of natural gas liquids. Results shown are fully risked estimates. For gas accumulations, all liquids are included as NGL (natural gas liquids). F95 represents a 95 percent chance of at least the amount tabulated; other fractiles are defined similarly. Fractiles are additive under the assumption of perfect positive correlation. TPS, total petroleum system; AU, assessment unit. Gray shading indicates not applicable]

\begin{tabular}{|c|c|c|c|c|c|c|c|c|c|c|c|c|c|}
\hline \multirow{3}{*}{$\begin{array}{c}\text { Total Petroleum Systems } \\
\text { (TPS) } \\
\text { and Assessment Units (AU) }\end{array}$} & \multirow{3}{*}{$\begin{array}{l}\text { Field } \\
\text { Type }\end{array}$} & \multicolumn{12}{|c|}{ Total Undiscovered Resources } \\
\hline & & \multicolumn{4}{|c|}{ Oil (MMBO) } & \multicolumn{4}{|c|}{ Gas (BCFG) } & \multicolumn{4}{|c|}{ NGL (MMBNGL) } \\
\hline & & F95 & F50 & F5 & Mean & F95 & F50 & F5 & Mean & F95 & F50 & F5 & Mean \\
\hline \multicolumn{14}{|c|}{ Upper Jurassic-Creataceous-Tertiary Composite TPS (50490100), continued } \\
\hline \multirow{2}{*}{$\begin{array}{l}\text { Austin-Tokio-Eutaw Updip Oil } \\
\text { and Gas AU (50490130) }\end{array}$} & Oil & 6 & 19 & 34 & 20 & 1 & 3 & 6 & 3 & 0 & 0 & 0 & 0 \\
\hline & Gas & & & & & 17 & 47 & 92 & 50 & 0 & 1 & 2 & 1 \\
\hline \multirow{2}{*}{$\begin{array}{l}\text { Austin-Eutaw Middip Oil and } \\
\quad \text { Gas AU (50490131) }\end{array}$} & Oil & 10 & 41 & 95 & 45 & 28 & 118 & 300 & 135 & 2 & 10 & 27 & 12 \\
\hline & Gas & & & & & 109 & 477 & 1,192 & 542 & 10 & 46 & 124 & 54 \\
\hline \multirow{2}{*}{$\begin{array}{l}\text { Austin Downdip Gas AU } \\
\quad(50490132)\end{array}$} & Oil & 3 & 11 & 32 & 13 & 10 & 43 & 130 & 53 & 1 & 4 & 14 & 5 \\
\hline & Gas & & & & & 406 & 1,429 & 3,013 & 1,542 & 46 & 167 & 385 & 185 \\
\hline $\begin{array}{l}\text { Post-Ouachita Successor Basin } \\
\text { AU (50490201) }\end{array}$ & Gas & \multicolumn{4}{|c|}{ Not quantitatively assessed } & & & & & & & & \\
\hline Triassic Basins AU (50490202) & Gas & \multicolumn{4}{|c|}{ Not quantitatively assessed } & & & & & & & & \\
\hline $\begin{array}{l}\text { Total Conventional } \\
\text { Resources }\end{array}$ & & 192 & 653 & 1,353 & 700 & 4,318 & 17,457 & 41,084 & 19,429 & 178 & 684 & 1,633 & 766 \\
\hline $\begin{array}{l}\text { Haynesville Sabine Platform } \\
\text { Shale Gas AU (50490161) }\end{array}$ & Gas & & & & & 44,268 & 59,735 & 80,604 & 60,734 & 22 & 35 & 55 & 36 \\
\hline $\begin{array}{l}\text { Haynesville Greater Gulf Basin } \\
\text { Shale Gas AU (50490162) }\end{array}$ & Gas & \multicolumn{4}{|c|}{ Not quantitatively assessed } & & & & & & & & \\
\hline $\begin{array}{l}\text { Mid-Bossier Sabine Platform } \\
\text { Shale Gas AU (50490163) }\end{array}$ & Gas & & & & & 2,879 & 4,870 & 8,240 & 5,126 & 5 & 10 & 18 & 10 \\
\hline $\begin{array}{l}\text { Bossier Greater Gulf Basin } \\
\text { Shale Gas AU (50490164) }\end{array}$ & Gas & \multicolumn{4}{|c|}{ Not quantitatively assessed } & & & & & & & & \\
\hline $\begin{array}{l}\text { Maverick Basin Pearsall Shale } \\
\text { Gas AU (50490165) }\end{array}$ & Gas & & & & & 3,386 & 7,764 & 17,801 & 8,817 & 0 & 0 & 0 & 0 \\
\hline $\begin{array}{l}\text { Greater Gulf Basin Lower } \\
\text { Cretaceous Shale Gas AU } \\
(50490166) \\
\end{array}$ & Gas & \multicolumn{4}{|c|}{ Not quantitatively assessed } & & & & & & & & \\
\hline $\begin{array}{l}\text { Eagle Ford Shale Oil AU } \\
(50490170)\end{array}$ & Oil & 341 & 758 & 1,687 & 853 & 625 & 1,486 & 3,533 & 1,707 & 12 & 29 & 74 & 34 \\
\hline $\begin{array}{l}\text { Eagle Ford Shale Gas AU } \\
(50490167)\end{array}$ & Gas & & & & & 23,470 & 46,150 & 90,747 & 50,219 & 851 & 1,809 & 3,842 & 2,009 \\
\hline $\begin{array}{l}\text { Austin Pearsall-Giddings Area } \\
\text { Oil AU (50490168) }\end{array}$ & Oil & 507 & 839 & 1,389 & 879 & 674 & 1,233 & 2,255 & 1,319 & 49 & 97 & 193 & 106 \\
\hline $\begin{array}{l}\text { Smackover Downdip } \\
\text { Continuous Gas AU (50490169) }\end{array}$ & Gas & \multicolumn{4}{|c|}{ Not quantitatively assessed } & & & & & & & & \\
\hline $\begin{array}{l}\text { Total Continuous } \\
\text { Resources }\end{array}$ & & 848 & 1,597 & 3,076 & 1,732 & 75,302 & 121,238 & 203,180 & 127,922 & 939 & 1,980 & 4,182 & 2,195 \\
\hline $\begin{array}{l}\text { Total Undiscovered } \\
\text { Oil and Gas Resources }\end{array}$ & & 1,040 & 2,250 & 4,429 & 2,432 & 79,620 & 138,695 & 244,264 & 147,351 & 1,117 & 2,664 & 5,815 & 2,961 \\
\hline
\end{tabular}


eastward into Texas and Louisiana, including the East Texas Basin Province (48), and continues to State waters and onshore lands primarily in Louisiana, Mississippi, Arkansas, and Alabama in the Louisiana-Mississippi Salt Basins Province (49). The TPS also includes Florida and small parts of Oklahoma, Missouri, Illinois, Kentucky, Tennessee, and Georgia (fig. 1). The Florida Peninsula Province (50) was not assessed as part of this study. The Upper Jurassic-Cretaceous-Tertiary Composite TPS contains 34 defined assessment units (AUs) for undiscovered conventional and continuous oil and (or) gas resources (table 1) within Jurassic and Cretaceous strata (fig. 2) that lie predominantly on the coastal plain and in State waters of Texas, Louisiana, Mississippi, Alabama, and a small part of the Florida panhandle.

\section{Resource Summary}

The USGS assessment of undiscovered conventional oil and gas resulted in estimated means of 19,429 billion cubic feet of gas (BCFG), 700 million barrels of oil (MMBO), and 766 million barrels of natural gas liquids (MMBNGL) in the AUs that were assessed (table 1). For undiscovered continuous oil and gas resources, the estimated means are 127,922 billion cubic feet of gas (BCFG), 1,732 million barrels of oil (MMBO), and 2,195 million barrels of natural gas liquids (MMBNGL) in the AUs that were assessed (table 1).

\section{For Additional Information}

Supporting geologic studies of Gulf Coast region total petroleum systems and assessment units are in progress, as well as studies of the methodology used in the assessment of both conventional and unconventional resources in Jurassic and Cretaceous strata of the Gulf Coast. Assessment results are available at the USGS Central Energy Resources Science Center Web site: http://energy.cr.usgs.gov/oilgas/noga.

\section{Gulf Coast Assessment Team}

Russell F. Dubiel (rdubiel@usgs.gov), Peter D. Warwick (pwarwick@usgs.gov), Sharon Swanson (sswanson@usgs.gov), Lauri Burke, Laura R.H. Biewick, Ronald R. Charpentier, James L. Coleman, Troy A. Cook, Kris Dennen, Colin Doolan, Catherine Enomoto, Paul C. Hackley, Alexander W. Karlsen, Timothy R. Klett, Scott A. Kinney, Michael D. Lewan, Matt Merrill, Krystal Pearson, Ofori N. Pearson, Janet K. Pitman, Richard M. Pollastro, Elizabeth L. Rowan, Christopher J. Schenk, and Brett Valentine.

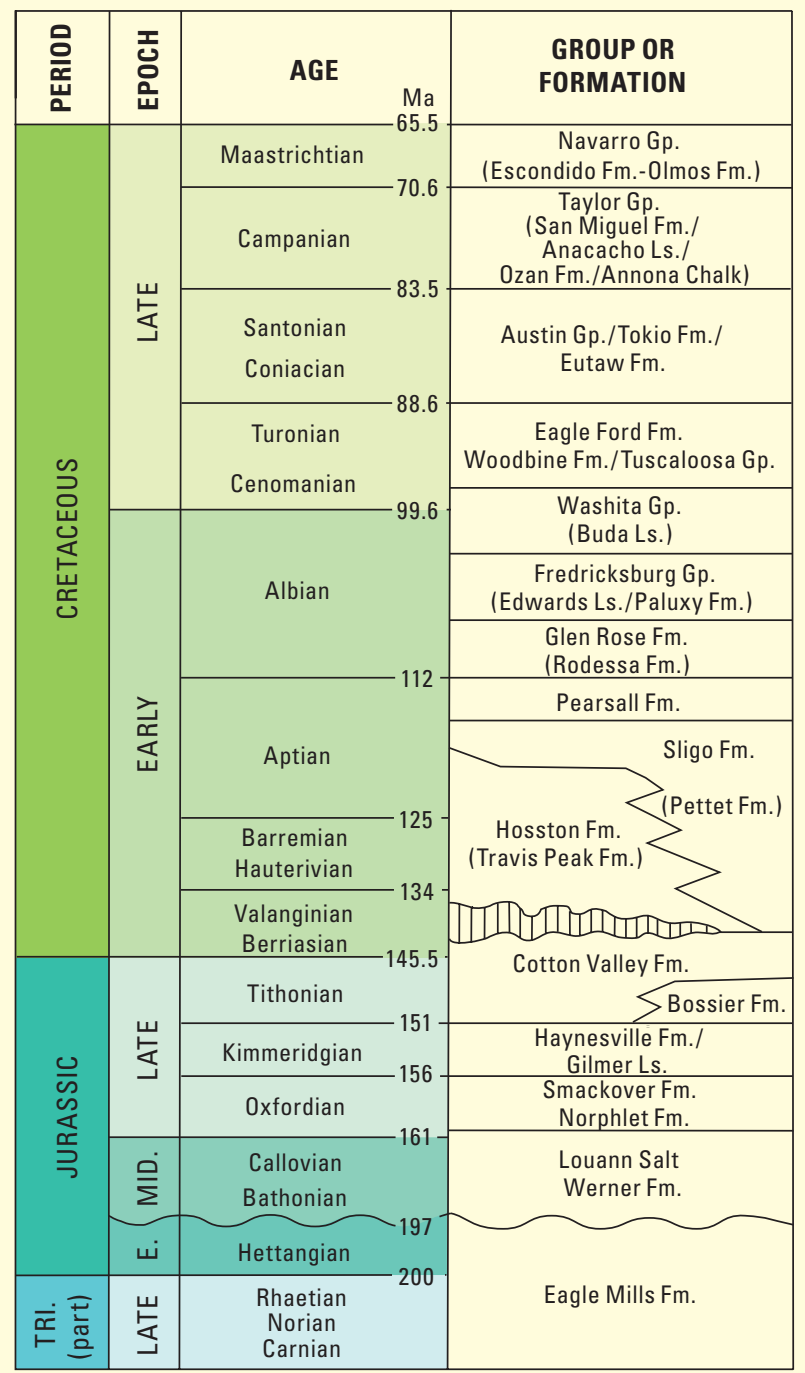

Figure 2. Generalized stratigraphic section showing geologic units for which petroleum resources were assessed in the Gulf Coast region. Nomenclature is a combination of formal and informal groups and formation and member names that are based on physical or biostratigraphic correlations in outcrop and subsurface studies. The nomenclature reflects the common designation and usage in the region by State, industry, U.S. Geological Survey, and academic geologists. (TRI., Triassic; E., Early; MID., Middle; Ma, million years ago; Fm., Formation; Gp., Group; Ls., Limestone). 